\title{
Physiological and Transcriptome Analyses Reveal the Response of Ammopiptanthus Mongolicus to Extreme Seasonal Temperature in Plateau Cold Desert Ecosystem
}

\section{Zimeng Yang}

Minzu University of China

Yiying Liu

Minzu University of China

Hang Han

Minzu University of China

Xinyu Zhao

Minzu University of China

\section{Siyu Chen}

Minzu University of China

Guofang Li

Minzu University of China

Sha Shi ( $\nabla$ shisha@muc.edu.cn )

Minzu University of China

Jinchao Feng

Minzu University of China

\section{Research Article}

Keywords: Ammopiptanthusmongolicus, Evergreen, Abiotic stress, Photosynthetic capacity, Transcriptome.

Posted Date: February 3rd, 2022

DOI: https://doi.org/10.21203/rs.3.rs-1265088/v1

License: (c) (i) This work is licensed under a Creative Commons Attribution 4.0 International License. Read Full License 


\section{Abstract}

Ammopiptanthus mongolicus is an evergreen broad-leaved shrub living in plateau cold desert ecosystem, which with remarkable tolerance to diverse abiotic stresses. We measured the photosynthetic physiological indexes of $A$.

mongolicus. under extreme seasonal climate in the field, and analyzed the transcriptome of its leaves. We found that the photosynthetic physiological indexes and gene expression at transcriptome level have significant characteristics when temperature changes. Under the low temperature condition, $A$. mongolicus. consumes excess light energy by increasing PSII non-regulatory energy dissipation $\mathrm{Y}(\mathrm{NO})$ at the physiological level. At the molecular level, it resists low temperature stress by changing photosynthesis, circadian rhythm and synthesis of unsaturated fatty acids, but its photosynthetic machinery is still damaged and photosynthesis is blocked. At the physiological level, $A$. mongolicus. regulates its own water content and improves the regulation of the PSIl regulatory energy dissipation Y(NPQ) to consumes excess light energy and to adapt to high temperature stress. At the molecular level, $A$. mongolicus. protects itself from high temperature and strong light by using the LHCII of PSII to consume excess energy, continuously assembling and maintaining PSII, and changing the production of antioxidants and so on.

\section{Introduction}

Ammopiptanthus mongolicus is a leguminous shrub, belonging to the third generation of ancient Mediterranean coastal plants. It is a remnant species that survives from the shrinking climate and drought in the ancient Mediterranean ${ }^{[1]}$. $A$. mongolicus. is mainly distributed in Inner Mongolia, Ningxia, Gansu and other provinces in China. It is one of the dominant species and constructive species of desert vegetation in Northwest China, and plays a decisive role in maintaining the stability of local ecosystem ${ }^{[2]}$. As an ancient relic species of the third season, $A$. mongolicus. has strong resistance to cold and drought. It can grow normally in the harsh environment with annual precipitation less than 200 $\mathrm{mm}$, evaporation of $2000-4000 \mathrm{~mm}$, extreme low temperature of $-24.8^{\circ} \mathrm{C}$ and extreme high temperature of $37.7^{\circ} \mathrm{C}$ [3] . A. mongolicus. is an ideal material for studying the stress resistance and adaptability of desert plants and has high scientific research value.

As the only ultra-xerophyte evergreen shrub in the arid region of Northwest China, researchers have studied the adaptation mechanism of $A$. mongolicus. to different temperatures. At the physiological level, some researchers found that $A$. mongolicus. adapts physiologically to maximize carbon assimilation during spring and summer, and to maximize foliar resistance to cold stress at the expense of photosynthesis in winter ${ }^{[3]}$. Some researchers found that under low temperature stress, the mitochondria of $A$. mongolicus. are more stable than chloroplasts, the number and volume of starch grains in chloroplasts decrease, and the fatty acid unsaturation of leaf lipids increases ${ }^{[4-6]}$ Under high temperature stress, the fatty acid unsaturation of $A$. mongolicus. is reduced ${ }^{[6]}$. At $50^{\circ} \mathrm{C}, A$. mongolicus. shows the characteristics of high temperature stress, photosynthetic electron transfer is blocked, and the photosynthetic apparatus can be restored after the stress condition is relieved ${ }^{[7]}$. In gene level research, some researchers looks for genes related to the adaptation of $A$. mongolicus. to different temperatures ${ }^{[8-10]}$, and establishes EST libraries of overwintering leaves

[11-12]. Some people have studied the expression of specific genes after stress at the transcriptome level ${ }^{[13-16]}$. However, few people combine physiology and transcriptome to explain the adaptation mechanism of $A$. mongolicus. from multiple angles and levels.

In this study, A. mongolicus. population which naturally grows in Etoke Banner, Western Ordos, Inner Mongolia is chosen as the research object. We use physiological research methods and transcriptome technology to study the characteristics of $A$. mongolicus. when temperature changes. It provides a scientific basis for people to better understand the adaptation mechanism and survival strategies of $A$. mongolicus. to changing temperature. 


\section{Results}

\section{Physiological characteristics of A. mongolicus. under different temperatures}

The photosynthetic physiological characteristics of $A$. mongolicus. show obvious differences under different seasonal temperature conditions (Fig. 1). Under low temperature conditions in winter, the average daily change of the net photosynthetic rate $(A)$ is negative, indicating that the daytime photosynthesis is lower than respiration and the growth of $A$. mongolicus. stops. Under high temperature condition in summer, the average daily $\mathrm{A}$ value increases by $82 \%$ compared with the control group, which proves that $A$. mongolicus. can accumulate materials normally under high temperature conditions.

The light energy utilization of $A$. mongolicus. is different under different temperature conditions (Fig. 2). In the low temperature condition, it accounts for a high proportion of energy for PSIl non-regulatory energy dissipation $Y(N O)$. $Y$ (NO) is an important indicator of light damage. Its high proportion indicates that the photochemical energy conversion and protective regulation mechanism cannot completely consume the light energy absorbed by the plant. At this time, the plant may have been damaged, or it may not be damaged at present but will be damaged if it continues to be illuminated. In the summer high temperature condition, most of the energy of $A$. mongolicus. is used for photosynthesis Y (II) and regulating energy dissipation Y (NPQ). The high value of Y (NPQ) indicates that the plants accept excess light, but it can still protect itself from damage by adjusting some processes (such as dissipating excess light energy into heat).

The effective photochemical quantum yield $\left(F_{v}{ }^{\prime} / F_{m}{ }^{\prime}\right)$ and the apparent electron transfer rate ETR are lower at low temperature than at high temperature (Fig. 3). It shows that low temperature significantly affects the primary electron capture efficiency and electron transfer efficiency of PS $\otimes$. The maximum photochemical quantum yield $\left(F_{v} / F_{m}\right)$ of PS $\nabla$ can characterize the photochemical efficiency of plants, and indicates the primary light energy conversion efficiency of

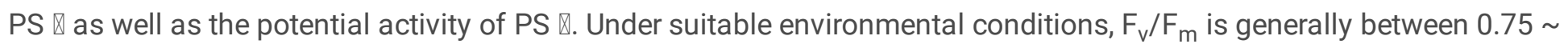
0.85. Under low temperature condition, the $\mathrm{F}_{\mathrm{v}} / \mathrm{F}_{\mathrm{m}}$ of $A$. mongolicus. is 0.32 , which is far lower than the normal value, indicating that the function of PS $\mathbb{Q}$ is damaged in the subzero low temperature environment. $F_{v} / F_{m}$ was 0.83 , which was in the normal range under both controlled condition and high temperature condition.

We conducted statistical analysis on the correlation of various photosynthetic parameters of $A$. mongolicus. at different temperatures. The results show that the net photosynthetic rate is highly correlated with intercellular $\mathrm{CO}_{2}\left(\mathrm{C}_{\mathrm{i}}\right)$ and leaf temperature $\left(T_{\text {leaf }}\right)$ under low temperature conditions, while the net photosynthetic rate is highly correlated with stomatal conductance $\left(g_{\mathrm{sw}}\right)$ and evaporation (E) under high temperature conditions (Tab. 1). 
Table 1

The correlation of photosynthetic parameters under differrent temperatures.

\begin{tabular}{|c|c|c|c|c|c|c|c|c|c|c|c|c|c|}
\hline \multirow[t]{2}{*}{$-7.5^{\circ} \mathrm{C}$} & \multicolumn{13}{|c|}{$28.7^{\circ} \mathrm{C}$} \\
\hline & A & $C_{i}$ & $T_{\text {leaf }}$ & Qin & $g_{s w}$ & E & & A & $C_{i}$ & $T_{\text {leaf }}$ & Qin & $g_{s w}$ & $E$ \\
\hline A & 1 & & & & & & A & 1 & & & & & \\
\hline$C_{i}$ & $-.927^{*}$ & 1 & & & & & $C_{i}$ & -0.219 & 1 & & & & \\
\hline $\mathrm{T}_{\text {leaf }}$ & $.906 *$ & -0.820 & 1 & & & & $T_{\text {leaf }}$ & -0.604 & -0.461 & 1 & & & \\
\hline Qin & 0.697 & -0.830 & 0.827 & 1 & & & Qin & -0.326 & -0.657 & $.934^{* *}$ & 1 & & \\
\hline$g_{s w}$ & -0.508 & 0.253 & -0.739 & -0.389 & 1 & & $g_{s w}$ & $.992^{* *}$ & -0.125 & -0.650 & -0.384 & 1 & \\
\hline E & -0.111 & -0.117 & -0.431 & -0.178 & $.905 *$ & 1 & E & $.968^{* k}$ & -0.383 & -0.393 & -0.105 & $.949^{* k}$ & 1 \\
\hline
\end{tabular}

\section{Transcriptome results of A. mongolicus. under different temperatures}

RNA-Seq sequencing results are good and meet the requirements of database construction. According to the functional classification of the differential gene detection results, it was found that the DEGs under low temperature stress were enriched in 2444 GO Terms. The DEGs under high temperature stress were enriched in 2207 GO Terms. The GO classification results of the two groups were similar, and most DEGs were related to molecular functions (Fig. 4, above).

In order to determine the changes in biochemical pathways of $A$. mongolicus. under different temperature conditions, the KEGG metabolic pathway analysis of the obtained Unigene found that under low temperature stress has 15 enrichment pathways, and under high temperature stress has 24 enrichment pathways (Fig. 4, below).

Under the low temperature condition, the circadian rhythm, photosynthesis, lipid metabolism, carbohydrate metabolism, plant hormone signal transduction and other metabolic pathways of $A$. mongolicus. are significantly enriched. In winter, the days are short and the nights are long. The circadian rhythm will affect the use of light energy of $A$. mongolicus., and low temperature will affect the activity of biological enzymes and thus affect the photosynthesis.

Under high temperature stress, metabolic pathways such as circadian rhythm, carbon fixation, carotenoid biosynthesis, secondary metabolites, cofactors and vitamin metabolism, lipid metabolism, amino acid metabolism and so on are significantly enriched. Studies have shown that when plants are subjected to environmental stresses such as high temperature and strong light, their photo-synthesis will be inhibited, leading to excess light energy. Excessive light energy will cause the plant to produce a large number of active oxygen free radicals, destroy the photosynthetic apparatus, and eventually cause photooxidation and even photodamage ${ }^{[17]}$. Among them, the metabolism of carotenoids, secondary metabolites, cofactors and vitamins are all related to the removal of reactive oxygen species.

Photosynthesis is an important physiological process of plants. Mapman's photosynthesis pathway includes four parts: photosynthetic phosphorylation, chloroplast respiration, Calvin cycle, and photorespiration. Under the low temperature

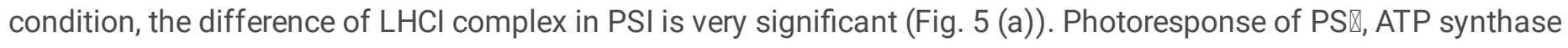
complex assembly factor (BFA3), subcomplex A during chloroplast respiration are all significantly different. Under the

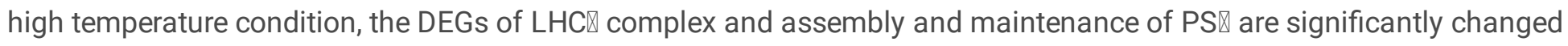


(Fig. 5 (b)). The DEGs assembled by Sedum heptulose-1,7-bisphosphatase (SBPase) and Rubisco enzyme were significantly enriched in Calvin cycle.

We used Pageman to analyze DEGs with Wilcoxon test. The results showed that: he photosynthetic phosphorylation of photosynthesis showed differences in low temperature condition (the front part of Fig. 6). PS $₫$ complex, PS $\otimes$ light response, $\mathrm{LHCl}$ complex, $\mathrm{PsaE}$ and chloroplast respiration showed differences in gene expression. The high temperature condition mainly affects the assembly and maintenance of PS区, and chloroplast respiration. The effect of phytohormones (the latter half of Fig. 6) is only significant in low temperature condition, among which: Jasmonic acid (JAs), auxin, brassinosteroids, cytokinins and signal peptides have all changed significantly. The abscisic acid (ABA), salicylic acid and cytokinin of $A$. mongolicus. vary greatly in high temperature environments.

\section{Discussion}

\section{DEGs under low temperature stress}

The results of field experiments showed that $A$. mongolicus. was damaged in the low temperature condition, $\mathrm{A}, \mathrm{Fv} / \mathrm{Fm}$ ', ETR as well as $F_{v} / F_{m}$ were reduced, $P S \otimes$ function was impaired, and photosynthetic capacity was weakened.

Compared with the control, PSII, Cyt $b_{6} f$ complex, PSI and ATPase showed differential gene expression in low temperature environment (Fig. 7). The inner peripheral antenna pigment proteins CP43 and CP47 of PSII bind to chlorophyll. They accept the excitation energy transferred from the surrounding antenna complex and transfer the energy to the reaction center complex. The changes of CP43 and CP47 will affect the absorption and transmission of light energy. In the PSII reaction center, light energy is converted into chemical energy. P680 absorbs light and is excited to become P680*, and then transfers electrons to pheophytin (Pheo). At the same time, the PSIl oxygen-evolving complex obtains electrons from the water molecules, which splits the water molecules and releases oxygen and protons. As one of the two core proteins that compose the reaction center complex, $D_{1}$ protein combines with various cofactors related to the original charge separation and electron transfer. $D_{1}$ protein plays an important role in the photosynthetic electron transfer process. Studies have found that low temperature can induce allosteric inactivation of $D_{1}$ protein, resulting in changes in the structure of thylakoid membranes and hindering electron transfer ${ }^{[18]}$. As part of the reaction center, $\mathrm{Cytb}_{559}$ can adjust the photoinhibition sensitivity of PS $\otimes$ through redox changes, so that the PS $₫$ reaction center is protected from damage ${ }^{[19]}$. The light energy absorption, energy conversion and electron transfer functions of PSII are impaired, resulting in a significant drop in $\mathrm{F}_{\mathrm{v}} / \mathrm{F}_{\mathrm{m}}$ far below the normal value. The results of Xiangchun Song are similar to those in this paper: the PS $₫$ reaction center of $A$. mongolicus. seedlings is irreversibly inactivated or the thylakoid membrane is damaged under subzero low temperature stress, which may produce serious photoinhibition. But Song believes that the peripheral antenna component of the optical system is more affected than the core complex at low temperature, which has not seen the corresponding results in this study ${ }^{[6]}$. Pheo transfers the received electrons to plastid quinone (PQ). PQ receives electrons and protons to form plastid hydroquinone $\left(\mathrm{PQH}_{2}\right)$. Then, the electrons of $\mathrm{PQH}_{2}$ are transferred to plastid cyanin (PC) on PSI through $\mathrm{Cyt}_{6} \mathrm{f}$, and hydrogen protons are released into the cavity of thylakoid to form a transmembrane proton gradient. Transmembrane proton gradient is the driving force for ATP synthesis. The change of $\mathrm{Cyt}_{\mathrm{b}} \mathrm{f}$ will not only affect the electron transfer function of photosynthesis, but also the synthesis of ATP.

The function of PSI is to transfer electrons from PC to ferredoxin for the reduction of NADP ${ }^{+}$. Recent studies have found that PSI is more sensitive to light and more prone to selective photoinhibition than PS $₫$ under low temperature and weak light conditions ${ }^{[20-21]}$. The results of KEGG analysis showed that the LHCI complex, PsaF and PsaE subunits of PSI showed differential gene expression. The main function of the light-harvesting pigment protein complex $\mathrm{LHCl}$ is to 
capture light energy. PsaF is a low-molecular-weight protein distributed in the membrane. Some studies believe that the $\mathrm{N}$-terminal amino acid sequence of eukaryotic PsaF is involved in the binding of PSI and PC ${ }^{[22]}$. PsaE, PsaD and PsaC together form the docking site of ferredoxin on the PSI receptor side ${ }^{[23-24]}$. Ferredoxin and ferredoxin-NADP ${ }^{+}$reductase in the photosynthetic electron transport chain are also affected, resulting in the hindrance of NADPH synthesis. The Ftype $\mathrm{H}^{+} / \mathrm{Na}^{+}$transport ATPase subunits also show differential expression of genes, which leads to impaired ATP synthesis. The low temperature affects the ability of absorbing light energy, transfering electrons, converting light energy into electric energy, and synthesizing NADPH as well as ATP, which ultimately leads to the decline of Fv'/Fm' and ETR and impaired photosynthesis capacity of $A$. mongolicus..

Compared with the light reaction, low temperature has a greater impact on the dark reaction. Because the dark reaction process is composed of many complex enzymatic reactions, and enzyme activity is very susceptible to temperature. KEGG results show that 13 related enzymes were differentially expressed in the dark reaction process. Rubisco enzyme is a key enzyme that determines the direction and efficiency of photosynthetic carbon metabolism in C3 plants, and is sensitive to temperature ${ }^{[25]}$. The results also show that the expression levels of 10 differentially expressed genes of Rubisco enzymes all declined. In Calvin cycle, only the gene expression of transketolase and glyceraldehyde-3phosphate dehydrogenase are not sensitive to temperature. In addition, the reduction phase of the dark reaction requires the use of NADPH and ATP produced by the light reaction. The inhibition of the synthesis of NADPH and ATP will inevitably affect the normal progress of the Calvin cycle.

Chloroplast respiration is an $\mathrm{O}_{2}$-dependent electron transport pathway in the chloroplast. Chloroplast respiration includes the non-photochemical reduction of $P Q$ by $N A D(P) H$ and the reoxidation of $P Q$ by terminal oxidase, which can consume excess electrons to protect plants from photooxidation damage. Ingo Ensminger studied the perennial evergreen woody Scots pine and found that the PSII reaction center of Scots pine is inactivated and the LHC structure changes under low temperature stress in winter. Its photosynthetic capacity will be restored when the low temperature stress is relieved ${ }^{[26]}$. Bauer et al. believe that perennial woody plants can resist low temperature stress by reducing photosynthesis and enhancing photoprotection mechanisms ${ }^{[27]}$. These may also be the adaptation strategies adopted by the evergreen shrub $A$. mongolicus. to resist low temperature.

Figure 8 shows the significantly enriched metabolic pathways of $A$. mongolicus under the low temperature conditions. There are three pathways related to carbohydrate metabolism that are significantly enriched. These three pathways are fructose and mannose metabolism, butanoate metabolism and C5-branched dibasic acid metabolism. The metabolism of fructose and mannose includes the ascorbic acid biosynthetic pathway. Ascorbic acid (ASA) also known as vitamin C, can be used as a cofactor of violaxanthin deepoxidase to participate in the lutein cycle, consume excess light energy and protect plants from harm.

Low temperature damages cell membranes first. Increasing the mass fraction of unsaturated fatty acids in the membrane is beneficial to improve the stability and fluidity of the membrane. Some studies show that the degree of unsaturation of fatty acid of the adult leaves of $A$. mongolicus. that grow naturally in the field is lower in summer and higher in autumn and winter ${ }^{[28]}$. In this study, the biosynthetic pathways of linoleic acid and arachidonic acid in $A$. mongolicus. were significantly enriched under the low temperature stress. Various proteins such as linoleate 13Slipoxygenase and cytochrome $\mathrm{P}_{450}$ family 2 subfamily $\mathrm{J}$ (CYP2J), which are involved in the metabolism of linoleic acid, shown differences in gene expression. Linoleate 13S-lipoxygenase is a common lipoxygenase in plants, which can catalyze the production of precursors of several important compounds including jasmonic acid. CYP2J is a group of $\mathrm{P}_{450}$ heme thiolate proteins, which are mainly distributed on the endoplasmic reticulum and the inner mitochondrial membrane, and it is involved in the synthesis of sterol hormones including brassinosteroids. Because light systems are distributed on the thylakoid membrane, damages to the membrane will affect the progress of plant photosynthesis. 
Hormones play an important role in plant resistance to adversity stress. Studies have shown that JAs has physiological functions such as inducing stomata closure, inhibiting photosynthesis, promoting respiration and promoting leaf senescence ${ }^{[29-30]}$. Treating plants with exogenous methyl jasmonate can induce the transcription of their heat shock protein family, increase the synthesis of antioxidants, reduce lipoxygenase activity and enhance the ability of plants to resist cold damage ${ }^{[31]}$.

\section{DEGs under high temperature stress}

Figure 9 shows the regulatory mechanism of $A$. mongolicus under the high temperature conditions. The mapman

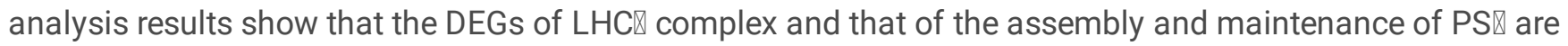
significantly changed. LHC II contains chlorophyll and carotenoids, which can capture and transmit light energy. Chlorophyll is an important photosynthetic pigment, which captures light energy and drives electrons to the reaction center. The chlorophyll molecule in the reaction center is related to photochemical quenching. The entire chlorophyll biosynthesis process (L-glutamyl-tRNA $\rightarrow$ chlorophyll $a \rightarrow$ chlorophyll $b$ ) involves 15 enzymes. The analysis found that 4/5 of the enzymes' expression genes have changed. Carotenoids include carotene and lutein, and their synthesis is affected by high temperature. Lutein participates in the lutein cycle, which can dissipate excess light energy and prevent membrane lipids from being peroxidized and thus maintain the stability of the thylakoid membrane structure and protect A. mongolicus. from high temperature stress and strong light stress.

The $D_{1}$ protein of the $P S \unrhd$ reaction center is rapidly degraded under strong light condition. In order to maintain the normal physiological needs of plants, the degraded $D_{1}$ protein will be replaced by the new $D_{1}$ protein produced by the repair mechanism. The reversible inactivation of the PS $₫$ reaction center can protect the photosynthetic system avoid destruction. This may be the reason for the significant change in the DEGs of assembly and maintenance of PSII.

Rubisco is the main site of high temperature inhibition of Calvin cycle ${ }^{[25]}$. KEGG analysis found that there were 7 (4 $\uparrow$, $3 \downarrow$ ) DEGs of Rubisco. The SBPase catalyzes the conversion of sedum heptulose-1,7-diphosphate (SBP) into sedum heptulose-7-phosphate (S7P) in the renewal phase. Same as under low temperature stress, only transketolase and glyceraldehyde-3-phosphate dehydrogenase remained unchanged in the Calvin cycle. In addition, NDH-mediated cyclic electron transfer may reduce the photooxidation damage caused by high temperature stress by shunting the excess electrons generated by the inhibition of $\mathrm{CO}_{2}$ assimilation to the chloroplast respiratory pathway [32]

Under the high temperature and strong light conditions, the net photosynthetic rates of the leaves showed two peaks on the curves of diurnal changes, and had obviously phenomena in midday depression of photosynthesis. The daily average $\mathrm{A}$ value was greater than that of the control group. These show that $A$. mongolicus. has a complete photosynthetic structure protection mechanism and can adapt to high temperature environments.

The pathway of significant enrichment related to carbohydrate metabolism under high temperature condition was the same as that of low temperature. Only the degree of enrichment of fructose and mannose metabolic pathways was higher in high temperature. Both C5-Branched dibasic acid metabolism and Butanoate metabolism were higher in the low temperature treatment.

Under high temperature and strong light conditions, the balance between the production and removal of reactive oxygen species (ROS) in plant cells was broken, and a large amount of reactive oxygen species accumulated in the cells. Active oxygen can cause the lipid peroxidation of the biomembrane, enlarge the pores of the membrane, increase the permeability, and affect the spatial structure of the enzymes on the membrane and thus leads to the destruction of chloroplasts. In severe cases, it will cause serious injury or even death to plants ${ }^{[33]}$. The gene expression of FabH and acetyl-CoA carboxylase (ACCase) during unsaturated fatty acid synthesis of A. mongolicus under high temperature stress was changed compared with that in April when temperature was suitable.

Page $7 / 19$ 
There are two types of active oxygen scavenging mechanisms in plants. (1) Enzymatic detoxification system: superoxide dismutase (SOD), ascorbate peroxidase (APX), catalase (CAT), etc. (2) Non-enzymatic antioxidants: ASA, carotenoids, glutathione, mannitol, flavonoids and so on ${ }^{[34]}$.

Secondary metabolites are the result of long-term adaptation of plants to their environment. They can improve the ability of plants to protect themselves, compete for survival, and coordinate the relationship between plants and the environment. Phenylpropane biosynthesis pathway is one of the three main secondary metabolic pathways in plants. It starts from phenylalanine and generates different phenylpropane metabolites through multi-step reactions, such as flavonoids, isoflavones, anthocyanins and Lignin and so on ${ }^{[35-36]}$. Anthocyanins can protect plants from light damage by quenching oxygen free radicals and reducing light energy absorption. Hughes studied 10 species of evergreen broadleaved trees and found that red leaves containing anthocyanins always maintained higher $F_{v} / F_{m}$ than green leaves. $\mathrm{F}_{\mathrm{v}}{ }^{\prime} / \mathrm{F}_{\mathrm{m}}$ ' is related to non-photochemical quenching. It means that trees with red leaves rely more on the light-damaged defense function of anthocyanins, rather than the light-damaged defense mediated by lutein [37].

Riboflavin is the precursor of flavin mononucleotide (FMN) and flavin adenine dinucleotide (FAD). As a prosthetic group of flavinase, FAD participates in multiple biochemical processes such as mitochondrial electron transport, photosynthesis, fatty acid oxidation and folate metabolism in plants ${ }^{[38]}$. Riboflavin can induce the accumulation of antioxidants in plant cells, and it can also promote plant growth by affecting the ethylene signaling pathway ${ }^{[39]}$. Biotin $\left(\mathrm{V}_{\mathrm{H}}\right.$ or $\left.\mathrm{V}_{\mathrm{B} 7}\right)$, as an essential cofactor for biotin-dependent carboxylase, plays an important role in life activities of plant. Common biotin-dependent carboxylase enzymes are: pyruvate carboxylase (PC) and ACCase. PC exists in the mitochondria and participates in the replenishment mechanism of the tricarboxylic acid cycle. ACCase plays a pivotal role in the feedback regulation of fatty acid synthesis and is the site of action for feedback regulation of fatty acid synthesis ${ }^{[40]}$.

The four pathways related to amino acid metabolism shown differences under high temperature, and the order of enrichment of each pathway was as follows: Valine, leucine and isoleucine biosynthesis>amino acid biosynthesis>lysine biosynthesis>glycine, serine and threonine metabolism. The branched chain amino acids valine, leucine and isoleucine and their derivatives are beneficial to plant growth and plant response to stress ${ }^{[41]}$. As an essential amino acid, the metabolism of lysine will affect many physiological reactions, such as: tricarboxylic acid cycle, abiotic and biotic stress response, starch metabolism, etc. ${ }^{[42]}$. Glycine, serine and threonine metabolic pathways combined with $\mathrm{GO}$ enrichment results shown that genes related to glycine catabolism and glycine dehydrogenation/decarboxylase activity have changed greatly. And it is known that when the activity of mitochondrial glycine decarboxylase increases, both photorespiration and photosynthesis will increase ${ }^{[43]}$.

In terms of hormones, salicylic acid, cytokinin, and abscisic acid (ABA) can improve plant active oxygen scavenging ability. Wang Xiaoling et al. believe that salicylic acid can reduce the damage of high temperature to seedlings by improving the ability of plants to resist oxidative stress and increasing the content of osmotic adjustment substances in cells ${ }^{[44]}$. Salicylic acid also has the function of delaying the degradation of $D_{1}$ protein and speeding up the recovery of $D_{1}$ protein after the high temperature is relieved ${ }^{[45]}$. Wang Feng et al. believe that ABA can improve the heat tolerance of plants by regulating the expression of heat-stress-induced genes at the transcriptional level ${ }^{[46]}$.

In conclusion, the photosynthetic physiological characteristics of $A$. mongolicus. have obvious temperature characteristics. Under the condition of low temperatures, the proportion of $Y(N O)$ increases, the function of PSQ is impaired and photosynthesis is hindered. Under the condition of high temperatures, $A$. mongolicus. maintains normal photosynthesis by adjusting gsw as well as water utilization and increasing the proportion of $\mathrm{Y}$ (NPQ). 
The gene expression of $A$. mongolicus. at the transcriptome level has obvious temperature changes, which mainly adapt to different temperature conditions by regulating genes related to molecular functions. A. mongolicus. resists the low temperature stress in autumn and winter by changing the circadian rhythm, increasing the synthesis of unsaturated fatty acids, and changing the action of plant hormones. However, the light energy absorption and electron transfer functions

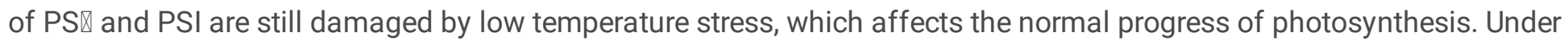
high temperature condition in summer, $A$. mongolicus. uses LHC II to consume excess energy, continuously assembles and maintains PS II, and changes the type of antioxidants, such as turning to synthesizing anthocyanins, flavonoids, and isoflavones, to protect itself from injury. In addition, porphyrin and chlorophyll metabolism, carotenoid metabolism, plant hormones, amino acid metabolism, unsaturated fatty acid synthesis and other metabolic pathways related differentially expressed genes have changed greatly.

\section{Materials And Methods}

\section{The research set}

Mengxi Town is located in Etuoke Banner, Ordos City, western Inner Mongolia $\left(106^{\circ} 43^{\prime}-108^{\circ} 54^{\prime} \mathrm{E}, 38^{\circ} 18^{\prime}-40^{\circ} 11^{\prime} \mathrm{N}\right)$, where is the natural distribution area of $A$. mongolicus. population. Climate type is typical temperate continental desert climate, which has the characteristics of large temperature difference between day and night, obvious seasonality, cold in winter and hot in summer, little rain, constant wind and sand. From 2010 to 2020 (2010.1-2020.12.31), the annual average temperature here is $10.3^{\circ} \mathrm{C}$, the lowest temperature is $-26.7^{\circ} \mathrm{C}$, and the highest temperature is $41.0{ }^{\circ} \mathrm{C}$; the average annual rainfall is only $120.4 \mathrm{~mm}$ (meteorological data source Reliable Prognosis; website https: https: //rp5.ru/docs/about/cn).

\section{Experimental Materials}

We selected April (mean temperature is $15.1^{\circ} \mathrm{C}$ ) as the control group, January (mean temperature is $-8.9^{\circ} \mathrm{C}$ ) as the lowtemperature control group and July (mean temperature is $26.8^{\circ} \mathrm{C}$ ) as the high-temperature control group. We selected the wild growing $A$. mongolicus. with a height of $90-100 \mathrm{~cm}$, a crown width of $100-150 \mathrm{~cm}$ and a base diameter of about $0.3 \mathrm{~cm}$ as the material. In January, April and July, we measured photosynthetic physiological indexes under natural conditions in the field. At the same time, we collected plant leaves and quickly placed them in liquid nitrogen for storage, and then transferred them to $-80^{\circ} \mathrm{C}$ refrigerator for storage for later use.

\section{Field experiment methods}

We used the Li-6800 portable photosynthetic instrument (LI-COR, Inc., Lincoln, NE, USA) to measure the diurnal changes of photosynthesis, and at the same time used the Li-6400XT portable photosynthetic instrument (LI-COR, Inc., Lincoln, NE, USA) to measure the diurnal changes of fluorescence. We were measured the diurnal changes on January 14, 2019 (daily average temperature: $-7.5^{\circ} \mathrm{C}$, highest temperature: $-2.1^{\circ} \mathrm{C}$, lowest temperature: $-13.1^{\circ} \mathrm{C}$ ) from 9:00-17:00, April 25 (daily average temperature: $16^{\circ} \mathrm{C}$, highest temperature: $25.8^{\circ} \mathrm{C}$, lowest temperature: $7.5^{\circ} \mathrm{C}$ ) and July 13 (daily average temperature: $28.7^{\circ} \mathrm{C}$, highest temperature: $35.2^{\circ} \mathrm{C}$, lowest temperature: $21.4^{\circ} \mathrm{C}$ ) from 7:00-19:00. The measurement is performed every two hours.

\section{Transcriptome experiment method}

BGI (Beijing) Co., Ltd. completed RNA extraction, library construction and sequencing of leaf slices of $A$. mongolicus.. The original data were processed, and the differentially expressed genes (DEGs) were screened for GO and KEGG gene function annotation analysis, Mapman analysis, Pageman analysis and qRT-PCT verification.

\section{Declarations}




\section{Funding}

This work was supported by National Natural Science Foundation of China (31570407), 2020 interdisciplinary research project of Central University for Nationalities (2020mdjc09) and Agricultural Cultural Heritage Protection and utilization project (2021xstd02).

\section{Author contributions}

Field experiment, Y.Z., L,Y., Z.X., C.S. and L.G.; Data processing, Y.Z. and H.H.; Resources, Z.X., C.S. and L.G.; Writingoriginal draft preparation, Y.Z. and L,Y.; Writing-review and editing, H.H., S.S. and F.J.;Supervision, S.S.All authors have read and agreed to the manuscript.

\section{Competing interests}

The authors declare no competing interests.

\section{Data Availability}

The data underlying this article are available in NCBI, and can be accessed with https://dataview.ncbi.nIm.nih.gov/object/PRJNA784165?reviewer=dh78oa7773uc8ua7d69197skqf .

Correspondence and requests for materials should be addressed to S.S.

\section{Statement}

We declare that we strictly abide by the guidelines of the IUCN Policy Statement on Research Involving Species at Risk of Extinction when collecting and using wild Ammopiptanthusmongolicus leaves in Ordos, Inner Mongolia, China. We used non-lethal sampling methods to collected 5-10 leaves from each healthy plant. The amount of leaf specimens we collected does not increase the extinction risk of $A$. mongolicus, and the research presented is critical to assist in the conservation of this species.

We have obtained the collection permit from the Administration of West Ordos National Nature Reserve in Ordos City. The collected leaf samples of $A$. mongolicus were stored in the Herbarium of the School of Life and Environmental Sciences, Minzu University of China (China) (temporarily placed in Laboratory 1103).

Species identifier: Liu Bo, Minzu University of China

\section{References}

1. Liu, M., Lu, C. and Yin, W. (2004) Research progress on the biological characteristics and stress resistance of Ammopiptanthusmongolicus. Chinese Journal of Applied and Environmental Biology. 03: 384-8.

2. Yue, G., Jin, M., Li, J., Yang, S., Guo, M. and Su, Y. (2015) Cloning and expression analysis of the proline transporter gene of Ammopiptanthusmongolicus. Biotechnology Bulletin. 31 (05): 106-112.

3. Li, H., Ma, W. and Li, H. (2000) Research on the stress resistance and development prospects of Ammopiptanthusmongolicus. World Forestry Research. 13(5): 67-71.

4. Han, S. and Wang, S. (2000) Electron microscopic observation of mitochondria in highly cold resistant plants in winter. Acta Botanica Boreali-OccidentaliaSinica. (04): 539-543.

5. Han, S., Zhang, H., Gu S., Wang, S. and Wang, Q. (2001) Starch grains of and their relationship with chloroplast development. Acta Botanica Boreali-OccidentaliaSinica. (01): 107-111. 
6. Song, X. (2009) Physiological Response on Photosynthesis with Seedlings of Two Ammopiptanthus (Leguminosae) Species to Low Temperature. Xinjiang University.

7. Zhang, M., Wang, H. and Yu, C. (2009) Dynamic characteristics of rapid chlorophyll fluorescence of super xerophytic plant Ammopiptanthusmongolicus. under high temperature stress. Journal of ecological environment. 18 (06): 2272-2277.

8. Liu, M., Lu, C., Shen, X. and Yin, W. (2006) Characterization and function analysis of a cold-induced AmCIP gene encoding a dehydrin-like protein in Ammopiptanthusmongolicus. Mitochondrial DNA. 17(5):342-349.

9. Liu, M., Shen, X., Yin, W. and Lu, C. (2006) Functional analysis of cold-inducible cDNA clones in the legume Ammopiptanthusmongolicus. Journal of Beijing Forestry University. 26(S2):180.

10. Liu, M., Shen, X. Lu,C. and Yin, W. (2007) Direct cloning of differential expression genes with full-length by a modified solid subtractive hybridization. Journal of Beijing Forestry University. (05): 67-72.

11. Guo, J., Sun, G., Shen, C., Qiao, H., Jia, L. and Guo, Z. (2007) Construction of Ammopiptanthusmongolicus. cDNA Library and the Primary Analysis of Expressed Sequence Tags(EST). Acta AgriculturaeBoreali-Sinica. (04):37-41.

12. Qiao, H., Wang, R., Guo J., Sun, G. and Li, G. (2009) The Cloning and Expression Vector of Ammopiptanthusmongolicus. Dehydrin Gene. Acta AgriculturaeBoreali-Sinica. 24(04):88-91.

13. Wen, X. (2015) Expression analysis of 15 candidate stress-resistant genes and functional identification of AmDREB1 in Ammopiptanthusmongolicus. Inner MongoliaAgriculturalUniversity.

14. Guo, T., Wang, M., Dong, B., Jiang, X. and Wu, Y. (2015) Cloning and expression analysis of AmDREB2C gene from Ammopiptanthusmongolicus. Journal of Plant Genetic Resources. 16(02):344-348.

15. Xue, M., Guo, T., Ren, M., Yin, Y. and Wang, M. (2018) Cloning, Transformation and Preliminary Function Analysis of AmFAD7 and AmFAD8 from Ammopiptanthusmongolicus. Acta Botanica Boreali-occidentaliaSinica. 38(08):13821391.

16. Pang, T., Ye, C., Xia, X. and Yin, W. (2013) De novo sequencing and transcriptome analysis of the desert shrub, Ammopiptanthusmongolicus. during cold acclimation using Illumina/Solexa. BMC genomics. 14(1): 488.

17. Georgios, L., Dimosthenis, N., Aspasia, K., Kornilios-Andrianos, V., Yiannis, M. and George, K. (2006) The photoprotective role of epidermal anthocyanins and surface pubescence in young leaves of grapevine (Vitis vinifera). Annals of botany., 98: 257-265.

18. Pospsil, P. and Taystjarvi, E. (1999) Molecular mechanism of high temperature-induced inhibition of acceptor side of photosystem II. Photosynthesis Research. 62:55-66.

19. Jia, H., Li, D., and Han, Y. (2001) Cytochrome b-559 in chloroplasts. Chinese Bulletin of Botany. (02):158-162.

20. Wang, Q., Wen, X. and Zhang, Q. (2003) Progress in Studies on photoinhibition. Chinese Bulletin Of Botany. 20 (5): 539-548.

21. Li, X., Duan, W., Meng, Q. and Zou, Q. (2002) PS $₫$ Photoinhibition Under Low Temperature. Plant Physiology Journal. 38 (4): 375-381.

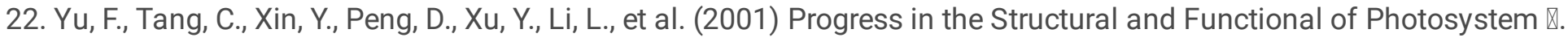
Chinese Bulletin of Botany. (03):266-275.

23. Anja, K-L., Ginga, S. and Pierre, S. (2020). Role of the two PsaE isoforms on $\mathrm{O}_{2}$ reduction at photosystem I in Arabidopsis thaliana. BBA - Bioenergetics.1861(1):

24. Niu, J., Wang, A., Zhu, H., Yang, L. and Li, Y. (2014) Cloning and expression analysis of sugarcane photosystem 『psaD gene.Journal of China Agricultural University. 19(01):43-50.

25. Hou, Z., Zhang, Y., Wang, H., Sun, K., Fang Z., Ma, D., et al. (2018) Effects of High-temperature Stress on Chlorophyll Mass Fraction and RuBP Carboxylase Gene Expression in Wheat (Triticum aestivum L.). Acta AgriculturaeBoreali- 
occidentalis Sinica. 27(09):1280-1286.

26. Ensminger, I., Sveshnikov, D., Campbell, D., Funk, C., Stefan, J. and Lloyd, J., et al. (2004) Intermittent low temperatures constrain spring recovery of photosynthesis in boreal Scots pine forests. Global change biology. 10(6):995-1008.

27. Bauer, H., Nagele, M., Comploj, M., Galler, V., Mair, M. and Unterpertinger, E. (1994) Photosynthesis in cold acclimated leaves of plants with various degrees of freezing tolerance. Physiologia Plantarum. 91(3):403-412.

28. Xue, M. (2019) Analyses of Leaf Membrane Lipid Composition and Chloroplast FAD gene Function in Ammopiptanthusmongolicus. under Different Stress Conditions. Inner Mongolia Agricultural University.

29. Dobritzsch, S., Weyhe, M., Schubert, R., Dindas, J., Hause, G. and Kopka, J., et al. (2015) Dissection of jasmonate functions in tomato stamen development by transcriptome and metabolome analyses. BMC Biology. 13:28.

30. Pan, R. and Li, H. (1989) Jasmonic acid-A natural growth inhibitor (in Chinese). Plant Physiology Communication. 2: $78-80$.

31. Li, M. and Yan, X. (2014) Jasmonic acid signaling in plants and its biological functions in relation to environment. Acta EcologicaSinica. 34 (23): 6779-6788.

32. Wang, P., Ye, J., Shen, Y. and Mi, H. (2006) Role of tobacco chloroplast NAD (P) H dehydrogenase in resistance to high temperature stress. Science in China Series C: Life Sciences, :109-117.

33. Chen, H., Wu, J. and Li, X. (2008) Production and scavenging mechanism of reactive oxygen species in chloroplasts. Progress in Modern Biomedicine, 2008(10):1979-1981.

34. Du, X. , Yin, W. , Zhao, Y. and Zhang, H. (2001) Production and scavenging mechanism of reactive oxygen species in plants. Chinese Journal of Biotechnology, 2001(02):121-125.

35. Wen, H. , Zhang, D. , Peng, C. , Wang, W. , Xie, X. and Rao, C. et al. (2017) Transcriptomic analysis of phenylpropane pathway in gastrodiaelata. Journal of Chinese Medicinal Materials, 40(4):789-796.

36. Wang, Y. , Yang, X. , Yang, R. , Wang, Y. , Yang, F. and Xia, P. et al. (2019) Research progress of MYB transcription factors regulating phenylpropane biosynthesis. Journal of Anhui Agricultural University, 46(05):859-864.

37. Hughes, N. and Smith, W. (2008) Seasonal photosynthesis and anthocyanin production in 10 broadleaf evergreen species. Functional Plant Biology, 34(12):1072-1079.

38. Sun, F. (2009) Function analysis of riboflavin receptor protein (RIR), RIR-like protein (AtRIR) and transcription factor RAP2.6L during defense response in Arabidopsis. Nanjing Agricultural University.

39. Taheri, P. and Tarighi, S. (2010) Riboflavin induces resistance in rice against Rhizoctonia solani via jasmonatemediated priming of phenylpropanoid pathway. Journal of Plant Physiology, 167(3):201-208.

40. Jiang, Y. and Tan, X. (2009) Research progress of plant acetyl-coa carboxylase gene in molecular biology. Nonwood Forest Research, 27(02):111-117.

41. Xing, A. and Last, R. (2017) A Regulatory Hierarchy of the Arabidopsis Branched-chain Amino Acid Metabolic Network. Plant Cell, 2017, 29, 1480-1499.

42. Yang, Q., Zhao, D. and Liu, Q. (2020) Connections Between Amino Acid Metabolisms in Plants: Lysine as an Example. Frontiers in Plant Science.

43. Timm, S., Florian, A., Arrivault, S., Stitt, M., Fernie, A. and Bauwe, H.(2012) Glycine decarboxylase controls photosynthesis and plant growth. FEBS Letters, 586(20):3692-3697.

44. Wang, X., Gao, Z., Yu, F., Wang, B. and Liu, T. (2011) Physiological response of exogenous salicylic acid to high temperature stress in Lupinus micranthusGuss. Chinese Agricultural Science Bulletin, 27 (25):89-93.

45. Zhao, H.,Zhao, X. ,Ma, P. , Wang, Y. , Hu, W. and Li, L. et al. (2011) Effects of salicylic acid on protein kinase activity and chloroplast $\mathrm{D}_{1}$ protein degradation in wheat leaves subjected to heat an high light stress. Acta Ecol Sin, 
31(5):259-263.

46. Wang, F., Cheng, F., Liu, Y., Zhong, L. and Zhang, G. (2006) Dynamic Changes of Endogenous Hormone content in Rice Grains at Different Temperature during Grain Filling stage. Acta Agron Sin, 2006(01):25-29.

\section{Figures}

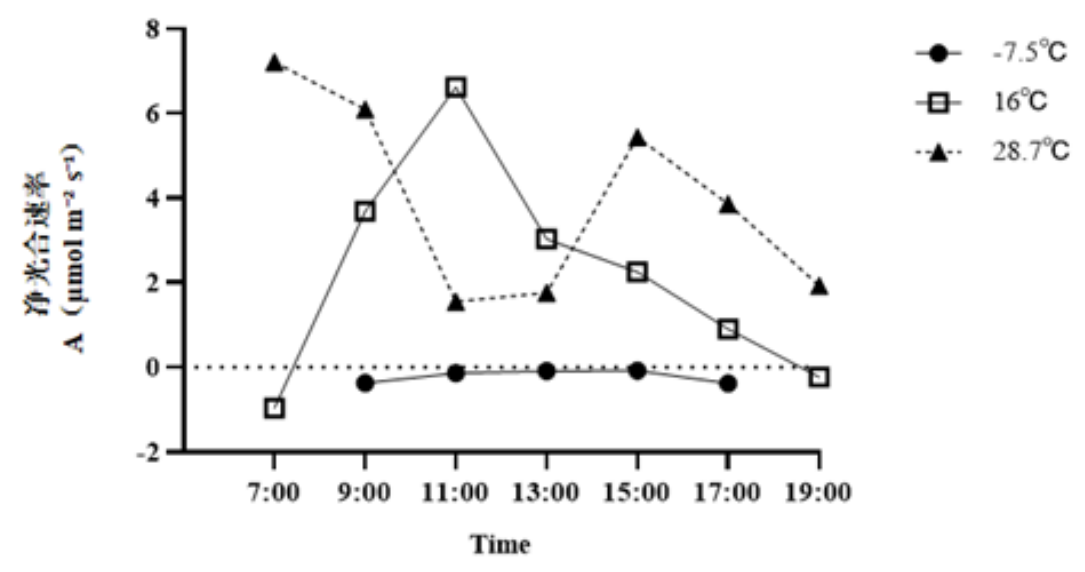

\section{Figure 1}

Daily changes of net photosynthetic rate (A) of $A$. mongolicus. under different temperatures
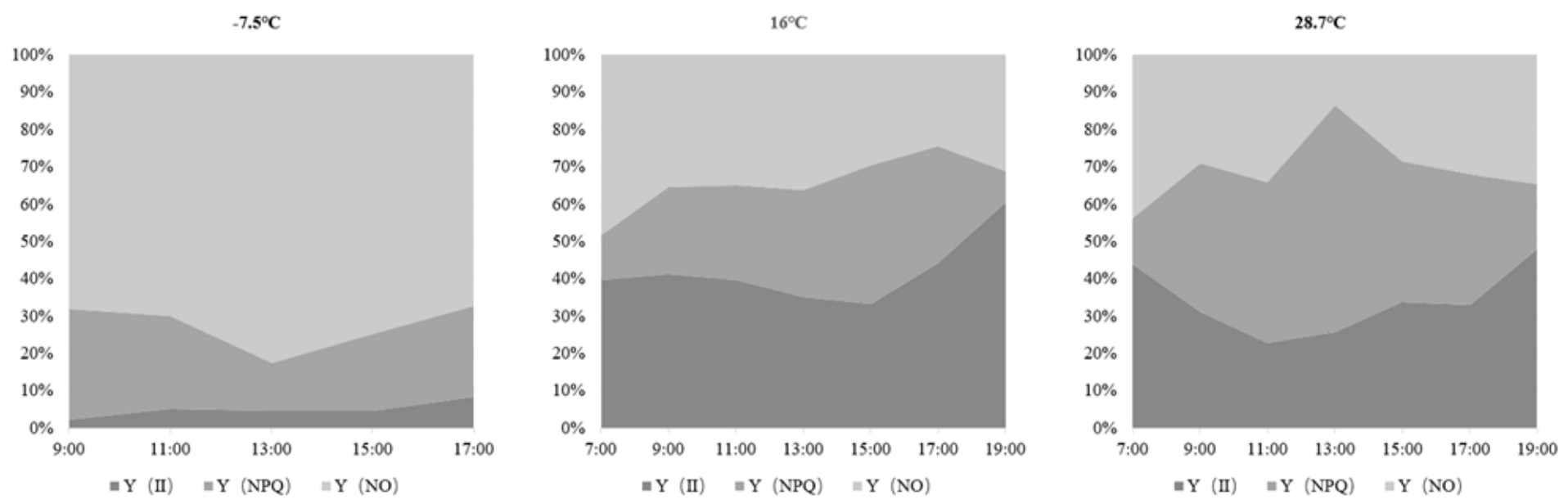

Figure 2

Light energy utilization under different temperatures. Y (II): energy used for photosynthesis. Y(NO): PSII non-regulatory energy dissipation. Y (NPQ): PSII regulatory energy dissipation. 

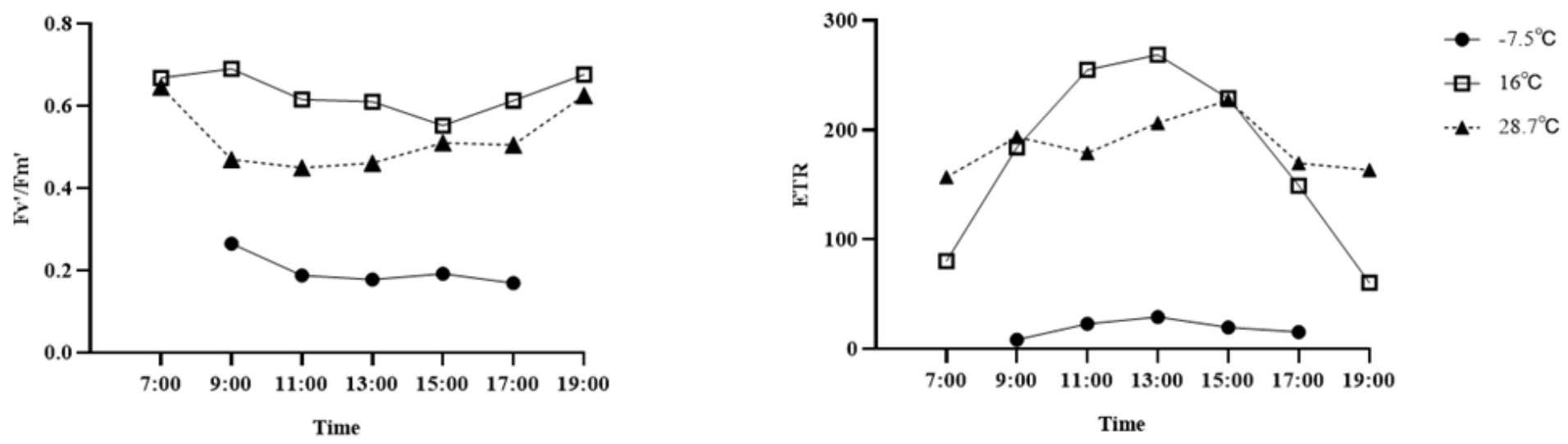

Figure 3

Diurnal changes of $\mathrm{F}_{\mathrm{v}}{ }^{\prime} / \mathrm{F}_{\mathrm{m}}{ }^{\prime}$ and ETR of $A$. mongolicus. under different temperatures. 


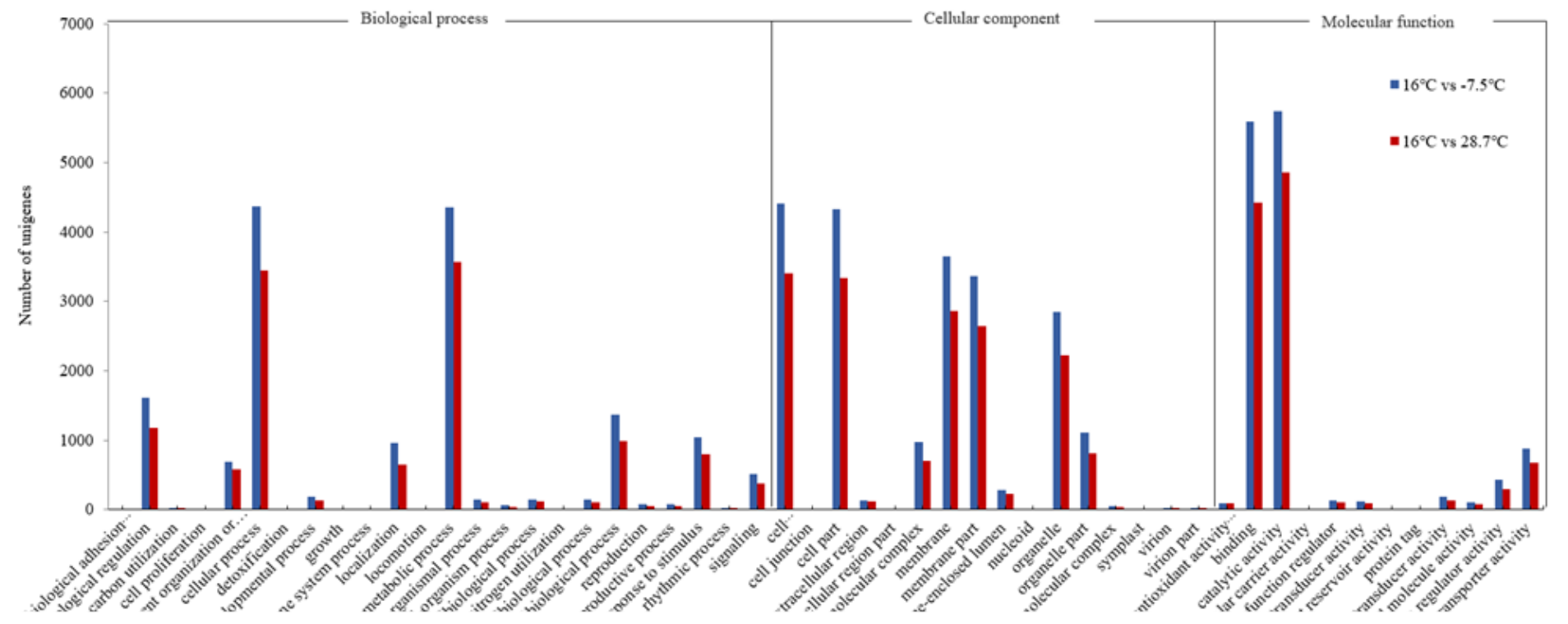

Figure 4

Gene Ontology (GO) functional classification analysis (above) and KEGG pathway analysis (below) of differentially expressed transcripts (DETs) based on RNA-Seq data. 


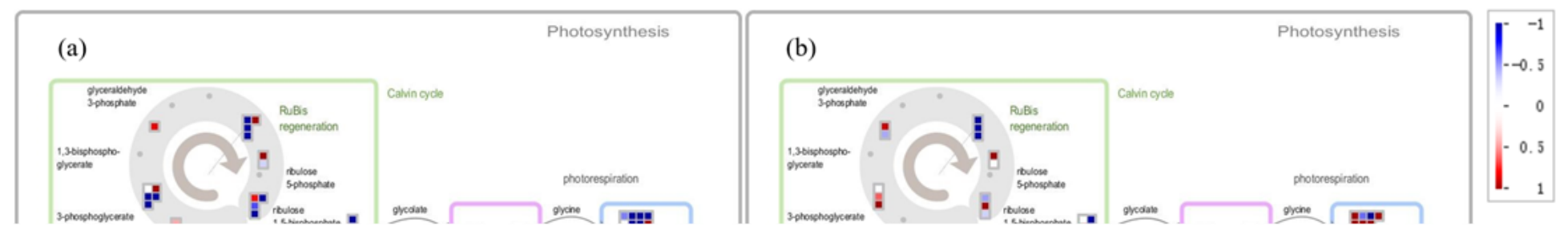

\section{Figure 5}

Differential gene expression in photosynthetic pathway under (a) low temperature stress (b) high temperature stress. The shade of the color indicates the level of differential expression multiples, and each square represents a differentially expressed gene. 
(a) (b)

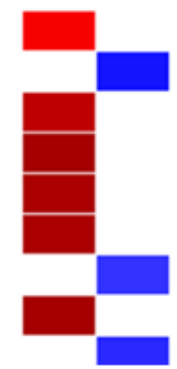

Photosynthesis

Photosynthesis. photophosphorylation. photosystem II. PS- II complex. reaction center complex

Photosynthesis. photophosphorylation. photosystem II. PS- II complex. reaction center complex. component CP47/PsbB

Photosynthesis. photophosphorylation. photosystem II. PS- II complex. Component PsbH

Photosynthesis. photophosphorylation. photosystem II. PS- II complex. Component PsbN

Photosynthesis. photophosphorylation. photosystem II. PS- II complex. Component PsbR

Photosynthesis. photophosphorylation. photosystem II. PS- II complex. Component PsbTc

\section{Figure 6}

Pageman analysis of differential gene expression in photosynthesis (above) and plant hormone action (below).Group (a) is the low temperature stress group, (b) is the high temperature stress group. The shade of the color indicates the level of differential expression multiples, and each square represents a differentially expressed gene. 


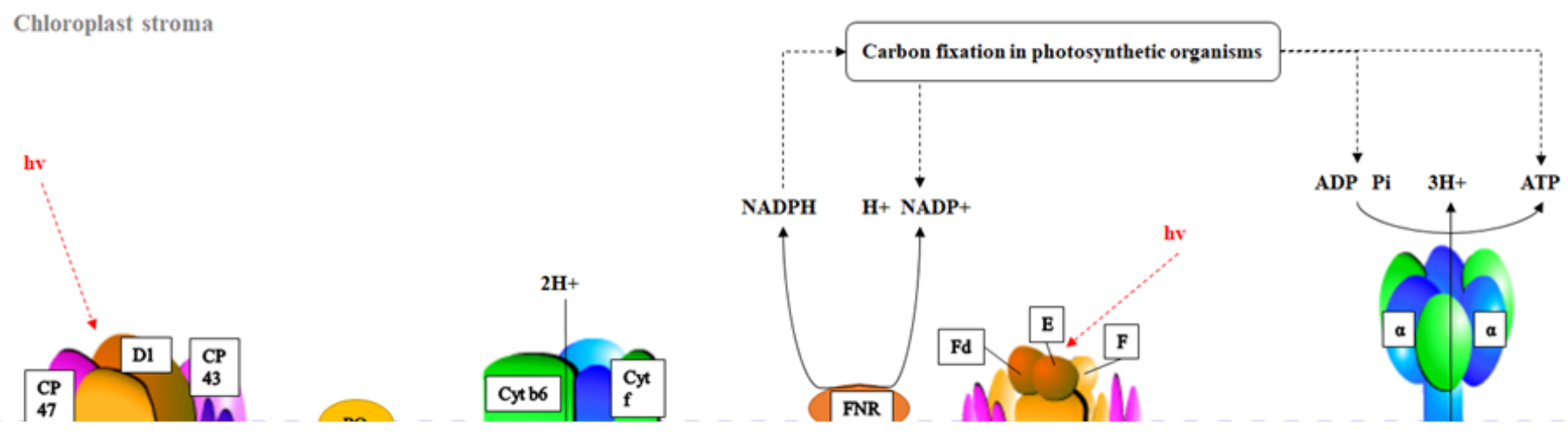

Figure 7

The photosynthesis response of $\boldsymbol{A}$. mongolicus. under low temperature stress. The part enclosed by the box indicates that this construct has differentially expressed genes under low temperature stress.

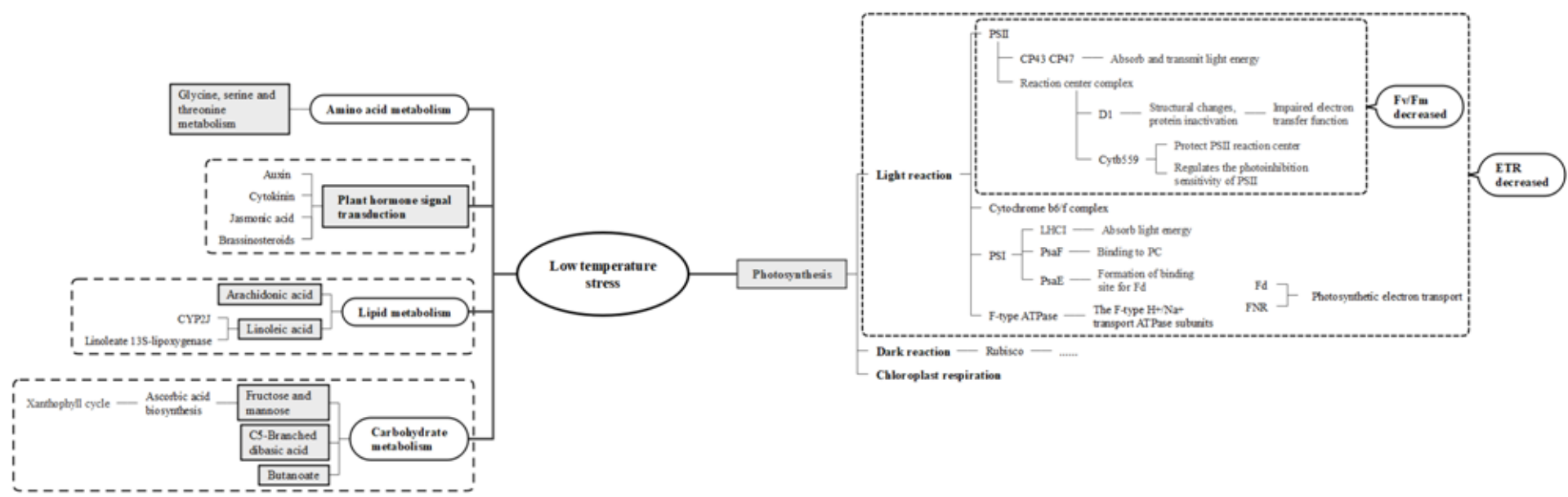

\section{Figure 8}

The regulation mechanism of $\boldsymbol{A}$. mongolicus under low temperature stress.Oval indicate the enriched metabolic pathways class. Rectangular representations represent enriched metabolic pathways. 


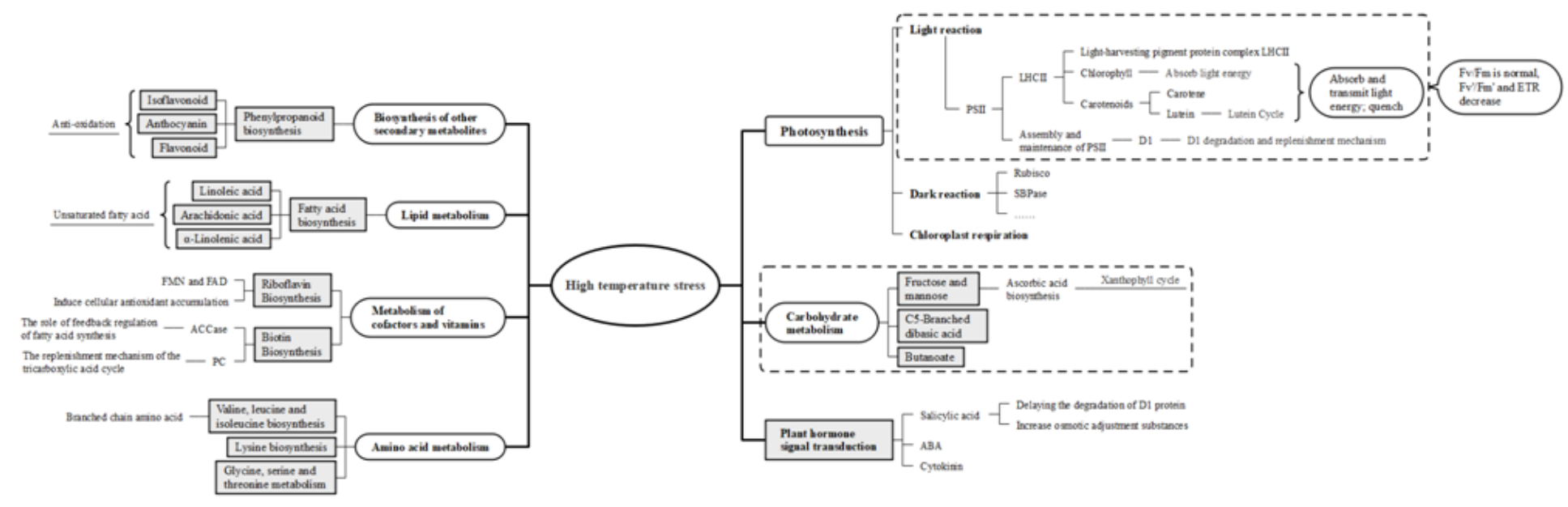

\section{Figure 9}

The regulatory mechanism of $\boldsymbol{A}$. mongolicus. under high temperature stress.Oval indicate the enriched metabolic pathways class. Rectangular representations represent enriched metabolic pathways. 